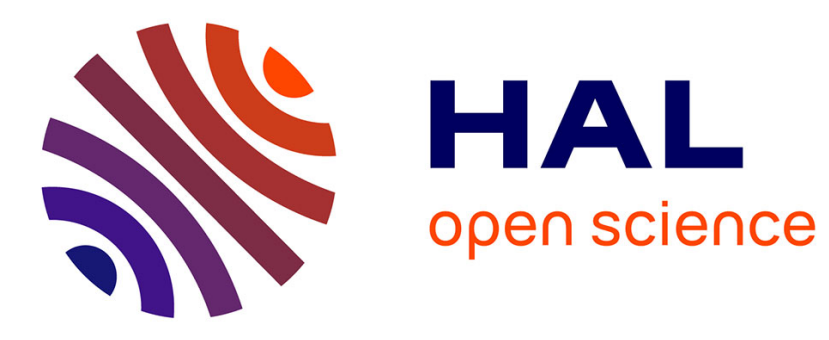

\title{
MAHEVE: An Efficient Reliable Mapping of Asynchronous Iterative Applications on volatile and Heterogeneous Environments
}

\author{
Raphaël Couturier, David Laiymani, Sébastien Miquée
}

\section{To cite this version:}

Raphaël Couturier, David Laiymani, Sébastien Miquée. MAHEVE: An Efficient Reliable Mapping of Asynchronous Iterative Applications on volatile and Heterogeneous Environments. HeteroPar'10, 8-th Int. Workshop on Algorithms, Models and Tools for Parallel Computing on Heterogeneous Platforms, 2011, Italy. pp.31-39. hal-00662874

\section{HAL Id: hal-00662874 https://hal.science/hal-00662874}

Submitted on 25 Jan 2012

HAL is a multi-disciplinary open access archive for the deposit and dissemination of scientific research documents, whether they are published or not. The documents may come from teaching and research institutions in France or abroad, or from public or private research centers.
L'archive ouverte pluridisciplinaire HAL, est destinée au dépôt et à la diffusion de documents scientifiques de niveau recherche, publiés ou non, émanant des établissements d'enseignement et de recherche français ou étrangers, des laboratoires publics ou privés. 


\title{
MAHEVE: An Efficient Reliable Mapping of Asynchronous Iterative Applications on Volatile and Heterogeneous Environments
}

\author{
Raphaël Couturier, David Laiymani, and Sébastien Miquée \\ University of Franche-Comté, LIFC laboratory, France \\ \{raphael.couturier, david.laiymani, sebastien.miquee\}@univ-fcomte.fr
}

\begin{abstract}
The asynchronous iteration model, called AIAC, has been proven to be an efficient solution for heterogeneous and distributed architectures. An efficient mapping of application tasks is essential to reduce their execution time. In this paper we present a new mapping algorithm, called MAHEVE (Mapping Algorithm for HEterogeneous and Volatile Environments) which is efficient on such architectures and integrates a fault tolerance mechanism to resist computing node failures. Our experiments show gains on a typical AIAC application execution time up to $65 \%$, executed on distributed clusters architectures containing more than 400 computing cores with the JaceP2P-V2 environment.
\end{abstract}

\section{Introduction}

In the parallel computing area, in order to execute very large applications on heterogeneous architectures, iterative methods are well adapted [2]. These methods repeat the same instructions block until a convergence state and a desired approximation of the solution are reached. They constitute the only known approach to solving some kinds of problems and are relatively easy to parallelize. The Jacobi or the Conjugate Gradient methods are examples of such methods. To parallelize them, one of the most used methods is the message passing paradigm which provides efficient mechanisms to exchange data between tasks. As such a method, we focus here on the asynchronous parallel iterative model, called AIAC (Asynchronous Iterations Asynchronous Communications).

In this model, as can be seen on Figure 1, after each iteration, a task sends its results to its neighbors and immediately starts the next iteration with the last received data. The receiving and sending mechanisms are asynchronous and tasks do not have to wait for the reception of dependency messages from their neighbors. Consequently, there is no idle time between two iterations. Furthermore, this model is tolerant to message loss and even if a task is stopped the remaining tasks continue the computation, with the last available data. Several experiments 2] show the relevance of the AIAC algorithms in the context of distributed clusters with high latency between clusters. These works underline the good adaptability of AIAC algorithms to network and processor heterogeneity.

In a previous study [6] we proposed the implementation of two static task mapping algorithms dedicated to the AIAC model on heterogeneous distributed 


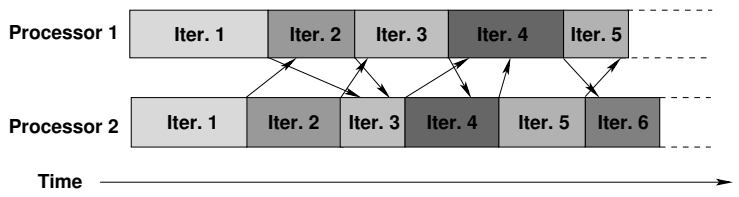

Fig. 1. Two processors computing in the AIAC model

clusters. Both these two algorithms, AIAC-QM (for AIAC Quick-quality Map) and F-EC (for Farhat Edges-Cuts) showed an important performance improvement by significantly reducing the application execution time. These experiments were performed by using the fully fault tolerant JaceP2P-V2 environment, described in the next section. In these experiments no computing node failures were introduced during the computation. As architecture heterogeneity continually evolves according to node volatility, we have to take care more precisely about the heterogeneity of the target platform. Thus in this paper we propose a new mapping algorithm called MAHEVE (Mapping Algorithm for HEterogeneous and Volatile Environments). This algorithm explicitly tackles the heterogeneity issue and introduces a level of dynamism in order to adapt itself to the fault tolerance mechanisms and to the evolution of the executing platform. Our experiments show gains up to $65 \%$ on application execution time, with faults during executions, which is about 10 points better than AIAC-QM and about 25 points better than F-EC, and MAHEVE also outperforms them in experiments with no fault during executions.

The rest of this paper is organized as follows. Section 2 presents the JaceP2PV2 middleware by describing its architecture and briefly presenting its fault tolerance mechanisms. Section 3 formalizes our mapping and fault tolerance problems and quotes existing issues to address them. Section 4 describes the new mapping strategy we propose, MAHEVE. In Section 5 we present the experiments we conducted on the Grid'5000 testbed with more than 400 computing cores. Finally, we give some concluding remarks and plan our future work in Section 6.

\section{JaceP2P-V2}

JaceP2P-V2 [5] is a distributed platform implemented in Java, dedicated to developing and executing parallel iterative asynchronous applications. It is fully fault tolerant allowing it to execute parallel applications over volatile environments. To our knowledge this is the only such existing platform.

The JaceP2P-V2 platform part, which is based on the daemons and supervisors paradigm, is composed of three main entities: the "super-nodes", which are in charge of supervising free computing nodes connected to the platform; the "spawner", which is launched by a user wanting to execute a parallel application. It is in charge of a group of computing nodes and monitors them. If one fails, it requires a replacing one to a super-node; the "daemon", first connects to a supernode and waits for a task to execute. Each daemon can communicate directly with its computing neighbors. 
To be able to execute AIAC applications, JaceP2P-V2 has an asynchronous messaging mechanism, and to resist daemon failures, it implements a checkpoint/restart mechanism by using a distributed backup mechanism called the uncoordinated distributed checkpointing [7]. This decentralized procedure allows the platform to be very scalable, with no weak point and does not require a secure nor a stable station for backups. When a daemon dies, it is replaced by another one, as we suppose that there are enough available free nodes. For more details on the JaceP2P-V2 platform, interested readers can refer to [5].

\section{Mapping and Fault Tolerance Problems}

Application modeling. The TIG [11] (Task Interaction Graph) model is the most appropriate to our problem, as it only models relationships between tasks. They are considered simultaneously executable and communications can take place at any time during the computation, with no precedence nor synchronization.

In this model, a parallel application is represented by a graph $G T(V, E)$, where $V=\left\{V_{1}, V_{2}, \ldots V_{v}\right\}$ is the set of $|V|$ vertices and $E \subset V \times V$ is the set of undirectional edges. Vertices represent tasks and edges represent the mutual communication among tasks. A function $E C: V \rightarrow \mathbb{R}^{+}$gives the computation cost of tasks and $C C: E \rightarrow \mathbb{R}^{+}$gives the communication cost for message passing on edges. We define $|V|=v, E C\left(V_{i}\right)=e_{i}$ and $C C\left(V_{i}, V_{j}\right)=c_{i j}$. Another function $D: V \rightarrow \mathbb{N}^{+}$gives the amount of dependencies of a task, noted $D\left(V_{i}\right)=d_{i}$.

Architecture modeling. A distributed clusters architecture can be modeled by a three-level-graph. The levels are architecture (a) (here the Grid'5000 grid), cluster (c), and computing node (n) levels. Let $G G(N, L)$ be a graph representing a distributed clusters architecture, where $N=\left\{N_{1}, N_{2}, \ldots N_{n}\right\}$ is the set of $|N|$ vertices and $L$ is the set of $|L|$ undirectional edges. The vertices represent the computing nodes and the edges represent the links between them. An edge $L_{i} \in L$ is an unordered pair $\left(N_{x}, N_{y}\right) \in N$, representing a communication link between nodes $N_{x}$ and $N_{y}$. A function $W N: N \rightarrow \mathbb{R}^{+}$gives the computational power of nodes and another function $W L: L \rightarrow \mathbb{R}^{+}$gives the communication latency of links. We define $W N\left(N_{i}\right)=w n_{i}$ and $W L\left(L_{i}, L_{j}\right)=w l_{i j}$. Let be $|C|$ the number of clusters contained in the architecture. A function $C N: C \rightarrow \mathbb{N}^{+}$ gives the amount of computing nodes contained in a cluster, and another function $C F: C \rightarrow \mathbb{N}^{+}$gives the amount of available computing nodes (not involved in computation) of a cluster. We define $C N\left(C_{i}\right)=C_{N i}$ and $C F\left(C_{i}\right)=C_{F i}$. We also define $C_{\bar{P} f i}$ as the average power of available resources of cluster $C_{i}$.

We evaluate the heterogeneity degree of the architecture, noted $h d$, by using the relative standard deviation method, with $h d=\frac{\sigma_{P N}}{a v g_{P N}}$ where $a v g_{P N}$ is the average computing power of nodes and $\sigma_{P N}$ represents the standard deviation of computing node power. This measure provides us the coefficient of variation of the platform in percentage - we only consider $0 \leq h d \leq 1$ as considering values of $h d>1$ is not relevant, as $h d=1$ denotes a fully heterogeneous platform. 
Mapping functions. When a parallel application App, represented by a graph $G T$, is mapped on a distributed clusters architecture, represented by a graph $G G$, the execution time of the application, $E T(A p p)$, can be defined as the execution time of the slowest task. Indeed, an application ends when all the tasks have detected convergence and reached the desired approximation of the solution. We define $E T(A p p)=\max _{i=1 \ldots v}\left(E T\left(V_{i}\right)\right)$, where the execution time of each task $i(i=1 \ldots v), E T\left(V_{i}\right)$, is given by $E T\left(V_{i}\right)=\frac{e_{i}}{w n_{i}}+\sum_{j \in J} c_{i j} \times w l_{i j}$ where $e_{i}$ is the computational cost of $V_{i}, w n_{i}$ is the computational power of the node $N_{i}$ on which $V_{i}$ is mapped, $J$ represents the neighbors set of $V_{i}, c_{i j}$ is the amount of communications between $V_{i}$ and $V_{j}$, and $w l_{i j}$ is the link latency between the computing nodes on which $V_{i}$ and $V_{j}$ are mapped. As described in this formula, the execution time of a task depends on the task weight and on the communications which may occur between this task and its neighbors. We underline here that in the AIAC model, it is impossible to predict the number of iterations of a task. So it is difficult to evaluate a priori its cost $e_{i}$.

An important point to take into consideration is that the execution of multiple tasks on the same node is not allowed, as this provides a fall of performance in such a context. This task mapping problem is similar to the classical graph partitioning and task assignment problem, and is thus NP-complete.

Fault tolerance. In volatile environments, computing nodes can disconnect at any time during the computation, and have thus to be efficiently replaced. The replacing nodes should be the best ones at the fault time, by finding them in available nodes. As executing environments can regularly evolve, due to computing node volatility, a mapping algorithm has to keep a correct overview of the architecture, in real time. Thus, criteria to assign tasks to nodes should dynamically evolve too.

Another problem appears after multiple crashes: some tasks may have migrated over multiple computing nodes and clusters, and the initial mapping may be totally changed. So, after having suffered some node failures the task mapping could not always satisfy the mapping criteria (not on the most powerful available machine, too far away from its neighbors...). A good fault tolerance policy has to evolve dynamically with the executing environment.

\subsection{Related Work}

In the literature of the TIG mapping many algorithms exist, which can be broadly classified into two categories. The first one is the Edge-cuts optimization class, which minimizes the use of the penalizing links between clusters. As tasks are depending on neighbors, which are called dependencies, the goal is to choose nodes where distance, in term of network, is small to improve communications between tasks. Here we can cite Metis [9] and Chaco [8] which are libraries containing such kind of algorithms. The second category is the Execution time optimization class, which aims at minimizing the whole application execution time. These algorithms look for nodes which can provide the smallest execution time of tasks using their computational power. We can cite QM [12] and MiniMax [10] as such kind of algorithms. Both classes of algorithms may fit with 
our goals as in our model we have both the computational power of nodes and communication costs which may influence the applications performance.

All mentioned algorithms do not tackle the computing node failures issue, or only basically by applying the same policy. As explained in Section 3. a more efficient and dedicated replacement function is needed. Nevertheless, to the best of our knowledge, no task mapping algorithm, addressing explicitly both the executing platform heterogeneity and the computing node failures issues, exists.

\section{MAHEVE}

Here we present our new task mapping strategy, called MAHEVE (for Mapping Algorithm for HEterogeneous and Volatile Environments). This algorithm aims at taking the best part of each category mentioned in Section 3.1, the edge-cuts minimization and the application execution time optimization algorithms.

This new algorithm can be divided into two parts. The first part aims at performing the initial mapping, and the second part is devoted to search replacing nodes when computing node failures occur.

\subsection{Initial Mapping}

In this section we will study the main mechanisms of the static mapping done by MAHEVE, which is composed of three phases: sort of clusters, sort of tasks, and the effective mapping, which maps tasks (in their sort order) on nodes of clusters (also in their sort order) with a reservation of some nodes in each cluster.

Sorting clusters. The first step of the initial mapping is to sort clusters according to the executing platform heterogeneity degree $h d$. The main principles are that a cluster obtains a better mark $M_{i}$ when $h d<0.5$ and it contains more computing nodes than other clusters $\left(C_{F i}\right.$, the number of available free nodes, is privileged), and when $h d \geq 0.5$ and it contains more powerful computing nodes $\left(C_{\bar{P} f i}\right.$, the average free computation power, is privileged). These choices come from several experiments with the AIAC model, which show that in such environments it is more efficient to privilege the computation power or the number of nodes. As the number of nodes, $C_{F i}$, and the average free computing power, $C_{\bar{P} f i}$, are not in the same order of magnitude, we normalize them with two functions, norm $N$ and normP. We note norm $N\left(C_{F i}\right)=N C_{F i}$ and $\operatorname{normP}\left(C_{\bar{P} f i}\right)=N C_{\bar{P} f i}$. The formula used to give a mark, $M_{i}$, to a cluster is $M_{i}=N C_{\bar{P} f i}^{h d}+N C_{F i}^{1-h d}(1)$.

This compromise function allows us to privilege clusters following our criteria, as explained previously, according to the heterogeneity degree. If we study its limits for the $h d$ extremities, $h d=0$ and $h d=1$, we obtain $\lim _{h d \rightarrow 0} M_{i}=$ $N C_{F i}+1$ and $\lim _{h d \rightarrow 1} M_{i}=N C_{\bar{P} f i}+1$, which fit with our objectives.

Clusters are so sorted and placed in a list containing them, starting from the cluster which receives the better mark to the one which receives the lower mark. 
Sorting tasks. Like clusters, tasks are also sorted according to the heterogeneity degree of the executing platform, $h d$. This sort is done in the same way as previously, as when $h d<0.5$ tasks with higher dependencies will be privileged, and when $h d \geq 0.5$ tasks with higher computing cost are privileged. The main function used to classified tasks is $Q_{i}=e_{i}{ }^{h d} \times d_{i}{ }^{1-h d}(2)$

where $Q_{i}$ is the evaluation of the task $i$ according to the heterogeneity degree $h d$ and $d_{i}$, the amount of dependencies of task $i$.

Then tasks are taken in the order of the first sort, determined with equation (2), and each task is placed in a new list (the final one) and some of its dependencies are added. We note $N b_{i}=d_{i}{ }^{1-h d}$ this amount of dependencies as the lower the heterogeneity degree is the higher this number will be. This final operation allows to control the necessary locality of tasks according to $h d$.

Mapping method. The third step of the initial mapping is to allocate tasks to nodes. As clusters and tasks have been sorted accordingly to the executing platform heterogeneity degree, ordered from the highest mark to the lowest, this function maps tasks on almost all available computing nodes of clusters, in their respective order in lists (for example a task classified first in the task list is mapped on an available node of the cluster classified first in the cluster list). The idea here is not to fulfill each cluster, but to preserve some computing nodes in each cluster. These conserved nodes will be used to replace failed nodes.

\subsection{Replacing Function}

During the initial mapping some nodes in each cluster have been preserved. When a node fails this function replaces it by a free node of the same cluster. If none is available this function sorts again clusters, to take into consideration platform modifications, and replaces the failed node by one available in the new sorted cluster list. This mechanism allows to retain task locality and a real time overview of the executing platform.

\section{Experimentation}

\subsection{A Typical AIAC Application and the Execution Platform}

We used a variation of the "Kernel CG" application of the NAS Parallel Benchmarks (NPB) 4] to evaluate the performance of our new mapping algorithm. The Conjugate Gradient method is replaced by the multisplitting method, which supports the asynchronous iterative model. More details about this method can be found in [3. We used used a matrix of size 5, 000, 000 with a bandwidth fixed to 35,000 , which generates between 8 and 20 neighbors per task. This application was executed on 64 nodes selected among more than 100.

The platform used to realize our tests, called Grid'5000 [1], is a French nationwide experimental set of clusters which provides us with distributed clusters architectures (28 heterogeneous clusters spread over 9 sites). We used three distributed clusters architectures, each having a different heterogeneity degree. 
Table 1. Application execution time in seconds and corresponding gains on various platforms using different mapping algorithms, with fault free (FF) executions and with 2 node failures each 20 seconds (WF) executions

\begin{tabular}{|c|c|c|c|c|c|c|c|c|}
\hline \multirow{2}{*}{$h d$} & Default & \multicolumn{2}{|c|}{ FT-AIAC-QM } & \multicolumn{2}{c|}{ FT-FEC } & \multicolumn{2}{c|}{ MAHEVE } \\
\cline { 2 - 8 } & FF & WF & FF & WF & FF & WF & FF & WF \\
\hline 0.08 & 80 & 229 & $63(21 \%)$ & $178(22 \%)$ & $61(23 \%)$ & $154(33 \%)$ & $60(25 \%)$ & $113(50 \%)$ \\
0.50 & 67 & 242 & $61(9 \%)$ & $118(51 \%)$ & $63(6 \%)$ & $133(45 \%)$ & $54(20 \%)$ & $85(65 \%)$ \\
0.72 & 67 & 192 & $59(12 \%)$ & $99(45 \%)$ & $65(3 \%)$ & $121(33 \%)$ & $52(22 \%)$ & $86(53 \%)$ \\
\hline
\end{tabular}

The first one was composed of four clusters spread over four sites, with a total of 106 computing nodes representing 424 computing cores with $h d=0.08$; the second one was composed of four clusters spread over three sites, with a total of 110 computing nodes representing 440 computing cores with $h d=0.50$; and finally the third one was composed of five clusters spread over four sites with 115 computing nodes representing 620 computing cores with $h d=0.72$.

All nodes can communicate with each other through an efficient network, but as it is shared with many other users, high latencies appear during executions.

\subsection{Experiments}

We compared MAHEVE with FT-AIAC-QM (for Fault Tolerant AIAC-QM) and FT-FEC (for Fault Tolerant F-EC) which are respectively the fault tolerant versions of the AIAC-QM and F-EC mapping algorithms presented in [6]. During some executions, we introduced two failures in computing nodes involved in the computation every 20 seconds to simulate a volatile environment. Table 1 shows the execution times of each mapping algorithm compared to the default mapping strategy of the JaceP2P-V2 platform, with the corresponding gains on application execution time, given in brackets. It presents both the executions with faults (WF) and the fault free (FF) ones.

First of all, we can note that all mapping algorithms provide an enhancement of the application performance by considerably reducing its execution time, especially for executions with node failures, with an average gain of about $45 \%$ in general in comparison to the default policy. If we focus on executions with node failures (WF), FT-FEC is efficient on architectures with a low heterogeneity degree $(h d=0.08)$ by providing gains of about $33 \%$, and gains are roughly the same on heterogeneous architectures $(h d=0.72)$. FT-AIAC-QM is efficient on architectures with a high heterogeneity degree $(h d=0.72)$ by providing gains of about $45 \%$, whereas it is not so efficient on homogeneous architectures $(h d=0.08)$ by providing gains of about $22 \%$. We can note here that on an architecture with a heterogeneity degree of 0.50 FT-AIAC-QM is more efficient than FT-FEC by providing gains up to $50 \%$. Here we point out that in fault free executions (FF), both algorithms also provide gains on their respective favorite architectures, though gains are lower than in executions with faults (WF).

Now if we focus on the performance of our new solution MAHEVE, we can see that it is all the time better than other algorithms. As can be seen in Table 1 in 
executions with faults (WF), it reduces the application execution time by about $50 \%$ on homogeneous architectures (here of 0.08 heterogeneity degree) which is more than 25 points better than FT-FEC and near 30 points better than FTAIAC-QM. On heterogeneous architectures (here of 0.72 heterogeneity degree) it also outperforms other mapping algorithms by reducing the application execution time by about $53 \%$ which is almost 10 points better than FT-AIAC-QM and 20 points better than FT-FEC. On middle heterogeneity degree architectures (here of 0.50 ), MAHEVE is once again better than its two comparative mapping algorithms by reducing the application execution time by about $65 \%$. These good performance come from the fact that it is designed to be efficient on both architectures, homogeneous and heterogeneous. Moreover, as it integrates a fault tolerance security in the initial mapping, it is more efficient when computing nodes fail. Here we can point out that this algorithm allows in general gains on application execution time of about $55 \%$. In fault free executions (FF), it outperforms once again the two other algorithms.

\section{Conclusion and Future Work}

In this paper we have presented a new mapping algorithm, called MAHEVE, to address the AIAC mapping issue on heterogeneous and volatile environments. It aims at doing an efficient mapping of tasks on distributed clusters architectures by taking the best part of the two known approaches, application execution time optimization and edge-cuts minimization. We have shown that it is all the time better than the two other comparative mapping algorithms, FT-AIAC-QM and FT-FEC. This can be explained by the fact that it not only takes care about computing nodes and clusters, but also about the task properties (computing cost and dependencies), what refines the mapping solution.

In our future work we plan to enhance the MAHEVE algorithm performance by modifying the notation of clusters, since their locality has not yet been taken into consideration, and enhanced fault tolerance functions should be tried. We also have to validate the algorithm performance with other AIAC applications.

\section{References}

1. Grid 5000, http://www.grid5000.fr

2. Bahi, J., Contassot-Vivier, S., Couturier, R.: Performance comparison of parallel programming environments for implementing AIAC algorithms. Journal of Supercomputing 35(3), 227-244 (2006)

3. Bahi, J., Contassot-Vivier, S., Couturier, R.: Asynchronous Iterations. In: Parallel Iterative Algorithms: from Sequential to Grid Computing. Numerical Analysis \& Scientific Computating, vol. 1. Chapman \& Hall/CRC, Boca Raton (2007)

4. Bailey, D., et al: The NAS Parallel Benchmarks. Tech. Rep. RNR-94-007, NASA Advanced Supercomputing (NAS) Division (March 1994)

5. Charr, J.C., Couturier, R., Laiymani, D.: JACEP2P-V2: A fully decentralized and fault tolerant environment for executing parallel iterative asynchronous applications on volatile distributed architectures. In: Abdennadher, N., Petcu, D. (eds.) GPC 2009. LNCS, vol. 5529, pp. 446-458. Springer, Heidelberg (2009) 
6. Couturier, R., Laiymani, D., Miquée, S.: Mapping asynchronous iterative applications on heterogeneous distributed architectures. In: PDSEC 2010 (2010)

7. Elnozahy, E.N., Alvisi, L., Wang, Y., Johnson, D.: A survey of rollback-recovery protocols in message-passing systems. ACM Comput. Surv. 34(3), 375-408 (2002)

8. Hendrickson, B., Leland, R.W.: The Chaco User's Guide (1995)

9. Karypis, G., Kumar, V.: A fast and high quality multilevel scheme for partioning irregular graphs. SIAM Journal on Scientific Computing 20(1), 359-392 (1998)

10. Kumar, S., Das, S.K., Biswas, R.: Graph partitioning for parallel applications in heterogeneous grid environments. In: IPDPS (2002)

11. Long, D.L., Clarke, L.A.: Task interaction graphs for concurrency analysis. In: ICSE, pp. 44-52 (1989)

12. Phinjaroenphan, P.: An Efficient, Pratical, Portable Mapping Technique on Computational Grids. Ph.D. thesis, RMIT University (2006) 